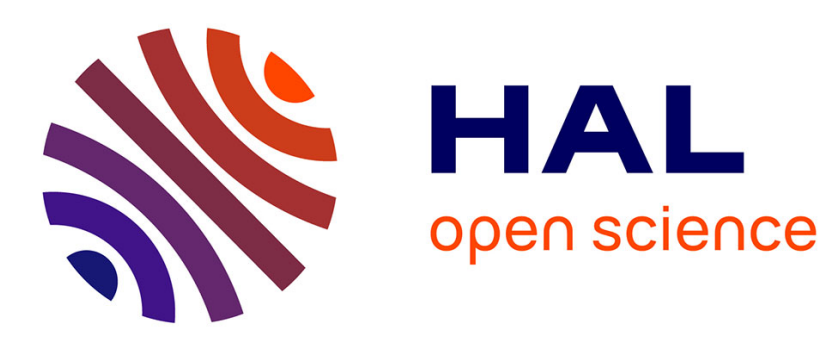

\title{
Fluorescence et absorption dans une fibre optique en PMMA dopé au crésyl violet
}

\author{
A. Saissy, Z.P. Cai
}

\section{To cite this version:}

A. Saissy, Z.P. Cai. Fluorescence et absorption dans une fibre optique en PMMA dopé au crésyl violet. Revue de Physique Appliquée, 1988, 23 (5), pp.941-946. 10.1051/rphysap:01988002305094100 . jpa00245896

\section{HAL Id: jpa-00245896 https://hal.science/jpa-00245896}

Submitted on 1 Jan 1988

HAL is a multi-disciplinary open access archive for the deposit and dissemination of scientific research documents, whether they are published or not. The documents may come from teaching and research institutions in France or abroad, or from public or private research centers.
L'archive ouverte pluridisciplinaire HAL, est destinée au dépôt et à la diffusion de documents scientifiques de niveau recherche, publiés ou non, émanant des établissements d'enseignement et de recherche français ou étrangers, des laboratoires publics ou privés. 


\title{
Fluorescence et absorption dans une fibre optique en PMMA dopé au crésyl violet
}

\author{
A. Saissy et Z. P. Cai \\ Laboratoire de Physique de la Matière Condensée (UA 190) Parc Valrose, 06034 Nice Cedex, France
}

(Reçu le 18 septembre 1987, révisé le 18 décembre 1987, accepté le 29 janvier 1988)

\begin{abstract}
Résumé. - Les bandes d'émission-absorption du crésyl violet diffusé dans une fibre optique en PMMA sont étudiées à $300 \mathrm{~K}$ et $77 \mathrm{~K}$. Les variations d'absorption sont décrites phénoménologiquement à l'aide d'une distribution gaussienne de facteurs d'absorption. Nous trouvons qu'entre $300 \mathrm{~K}$ et $77 \mathrm{~K}$, le maximum de la distribution se déplace de $-233 \mathrm{~cm}^{-1}$ tandis que son amplitude augmente de $34 \%$. Une augmentation du coefficient de transmission avec l'éclairement est mise en évidence dans une fibre faiblement dopée $\left(10^{-4} \mathrm{M} / \mathrm{l}\right)$ refroidie à $77 \mathrm{~K}$.
\end{abstract}

\begin{abstract}
We study emission-absorption bands of cresyl violet diffused PMMA optical fiber for $300 \mathrm{~K}$ and $77 \mathrm{~K}$ temperatures. Absorption variations are described by a Gaussian distribution of absorption coefficients. We found $\mathrm{a}-233 \mathrm{~cm}^{-1}$ shift for the maximum distribution with $34 \%$ increase of amplitude. The transmission coefficient of a weakly doped fiber $\left(10^{-4} \mathrm{M} / \mathrm{l}\right)$ at $77 \mathrm{~K}$ increase with intensity.
\end{abstract}

\section{Introduction.}

Les polymères chargés de colorant constituent des matériaux susceptibles de multiples applications en optique [1, 2]. Récemment, nous avons étudié le dopage de fibres optiques en polymère par diffusion de colorants [3]. Ainsi sont obtenues des structures du type guide d'onde de lumière présentant des bandes d'absorption-émission intenses sur un large domaine spectral. Les dimensions longitudinale et transversale de ces guides permettent d'envisager, à partir de sources lumineuses de puissance modeste, la réalisation d'expériences d'amplification, d'absorption saturée, etc... réservées jusqu'ici aux solutions liquides de ces colorants. Une première étape dans cette direction est la connaissance de la nature exacte des bandes d'absorption-émission d'un colorant placé dans un polymère.

Cette note est consacrée à l'étude des bandes d'absorption-émission du crésyl violet diffusé dans des fibres optiques en polyméthylméthacrylate (PMMA). Ces bandes sont caractérisées par leurs spectres à la température ambiante et à l'azote liquide. Les variations d'amplitude, de position et de largeur en fonction de la température sont décrites qualitativement et quantitativement à l'aide d'un modèle de bandes à profil gaussien. En dernier lieu, une étude à la température de l'azote liquide de la transmission d'une fibre dopée avec la puissance lumineuse nous a permis de mettre en évidence un comportement non linéaire de ces fibres.

\section{Variation des bandes d'émission-absorption avec la température.}

Les mesures sont effectuées sur des fibres optiques à saut d'indice de diamètre de cœur $250 \mu \mathrm{m}$ et de gaine optique très mince. La gaine optique est en polyéthylène, le cœur en PMMA pur. Le dopage de cette fibre avec du crésyl violet est réalisé par diffusion du colorant lors de l'immercion de la fibre dans une solution de crésyl violet dan, du méthanol pour les concentrations $10^{-4}$ et $10^{-3} \mathrm{M} / \mathrm{l}$. La durée de la diffusion est de $22 \mathrm{~h}$, la longueur de la fibre est de $1 \mathrm{~m}$.

L'utilisation d'un laser $\mathrm{HeNe}$ réglé sur la raie $6328 \AA$, de puissance $25 \mathrm{~mW}$, nous a conduit à choisir comme colorant le crésyl violet pour son absorption à cette longueur d'onde. Le faisceau lumineux émis par le laser est focalisé sur la face d'entrée de la fibre avec une lentille de $2 \mathrm{~cm}$ de distance focale, la lumière de fluorescence émergeant de la fibre est analysée spectralement avec un monochromateur à réseau Jobin-Yvon M25.

Le spectre de fluorescence à $77 \mathrm{~K}$ est étudié en plongeant directement la fibre dans de l'azote 
liquide. Les figures 1 et 2 représentent ces spectres pour des fibres dopées à partir de solutions de crésyl violet à $10^{-3}$ et $10^{-4} \mathrm{M} / \mathrm{l}$.

Pour la fibre à $10^{-4} \mathrm{M} / 1$, nous observons que la transmission à $6328 \AA \AA$ est nulle à $300 \mathrm{~K}$ mais devient mesurable à $77 \mathrm{~K}$, il se produit donc une variation de la transmission de la fibre entre $300 \mathrm{~K}$ et $77 \mathrm{~K}$. La même expérience réalisée avec une fibre non dopée ne nous a pas révélé de variation importante de la transmission, cette variation provient donc bien du colorant.

Une étude du spectre de ces variations de transmission a été réalisée avec une lampe à filament de tungstène de faible puissance. Les fibres sont dopées sur $10 \mathrm{~cm}$, à mi-longueur, avec des solutions à $10^{-3}$ et $10^{-4} \mathrm{M} / 1$, la durée de la diffusion est $17 \mathrm{~h}$. La figure 3 représente les spectres des variations relatives de l'intensité transmise par la fibre soit

$$
\frac{\Delta I}{I}=[I(77)-I(300)] / I(300) \text {. }
$$

Posons $I(T)=I_{0} \exp -\alpha_{T} L$ avec $\alpha_{T}$ coefficient d'absorption de la fibre à la température $T$, alors $L \Delta \alpha_{T}=\log (1+\Delta I / I)$. Lorsque $\Delta I \ll I$ nous

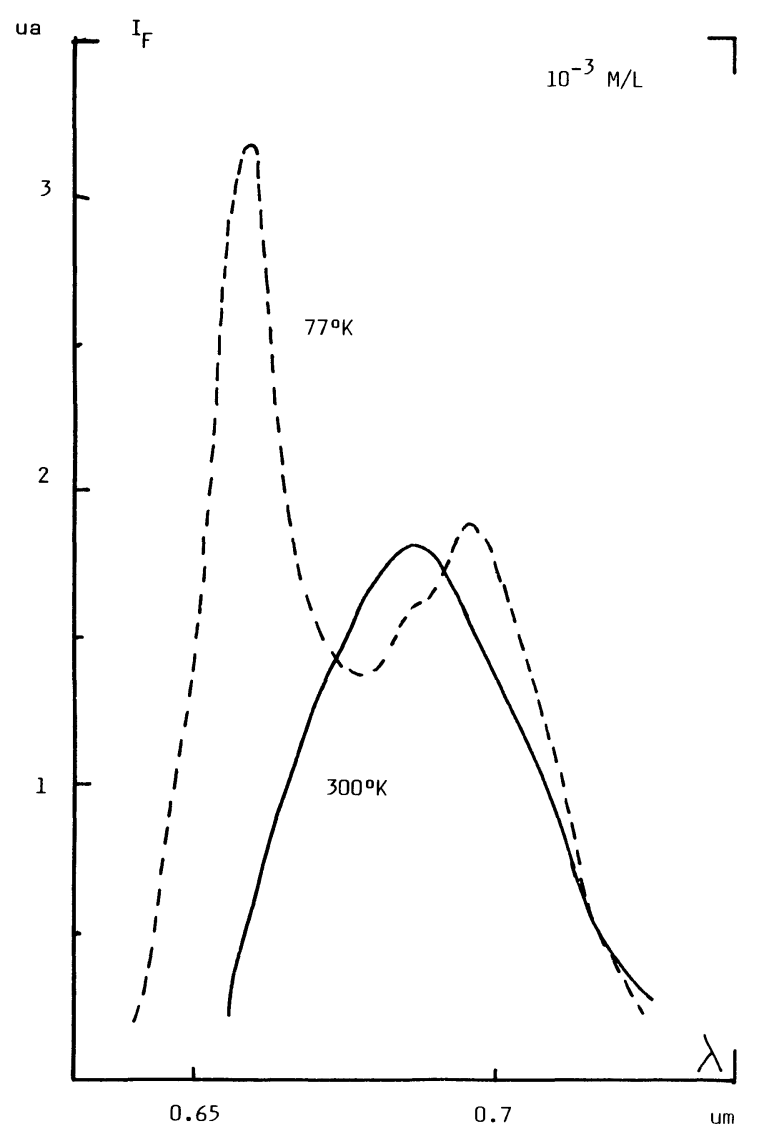

Fig. 1. - Spectres de fluorescence d'une fibre dopée à $10^{-3} \mathrm{M} / 1$ pendant $22 \mathrm{~h}$ : $-300 \mathrm{~K} ;----77 \mathrm{~K}$.

[Fluorescence spectra of $10^{-3} \mathrm{M} / 1$ doped fiber, diffusion time $=22 \mathrm{~h}$ : $300 \mathrm{~K} ;----77 \mathrm{~K}$. pouvons utiliser un développement limité du logarithme soit $\Delta \alpha_{T} \approx \frac{\Delta I}{L I}$.

Les variations d'absorption et d'émission en fonction du nombre d'onde $\sigma=1 / \lambda$ sont portées sur la figure 4 . On constate que le symétrique du spectre de la variation d'émission par rapport à la verticale : $\sigma=15500 \mathrm{~cm}^{-1}$ reproduit les variations de l'absorption, c'est-à-dire qu'à une augmentation de l'absorption correspond une augmentation de l'émission et réciproquement. L'expérience nous confirme donc que la loi dite du miror image reliant les spectres d'absorption et d'émission est bien vérifiée par nos mesures ; ceci est d'autant plus satisfaisant que les mesures d'absorption et d'émission ont été réalisées sur deux séries de fibres dopées indépendamment. Absorption et émission étant liées, notre interprétation portera uniquement sur les variations de l'absorption avec la température.

Assimilons chaque molécule de colorant à un petit oscillateur de coefficient d'absorption $\Gamma_{0}$ dans un repère lié à l'oscillateur. Du fait de l'agitation

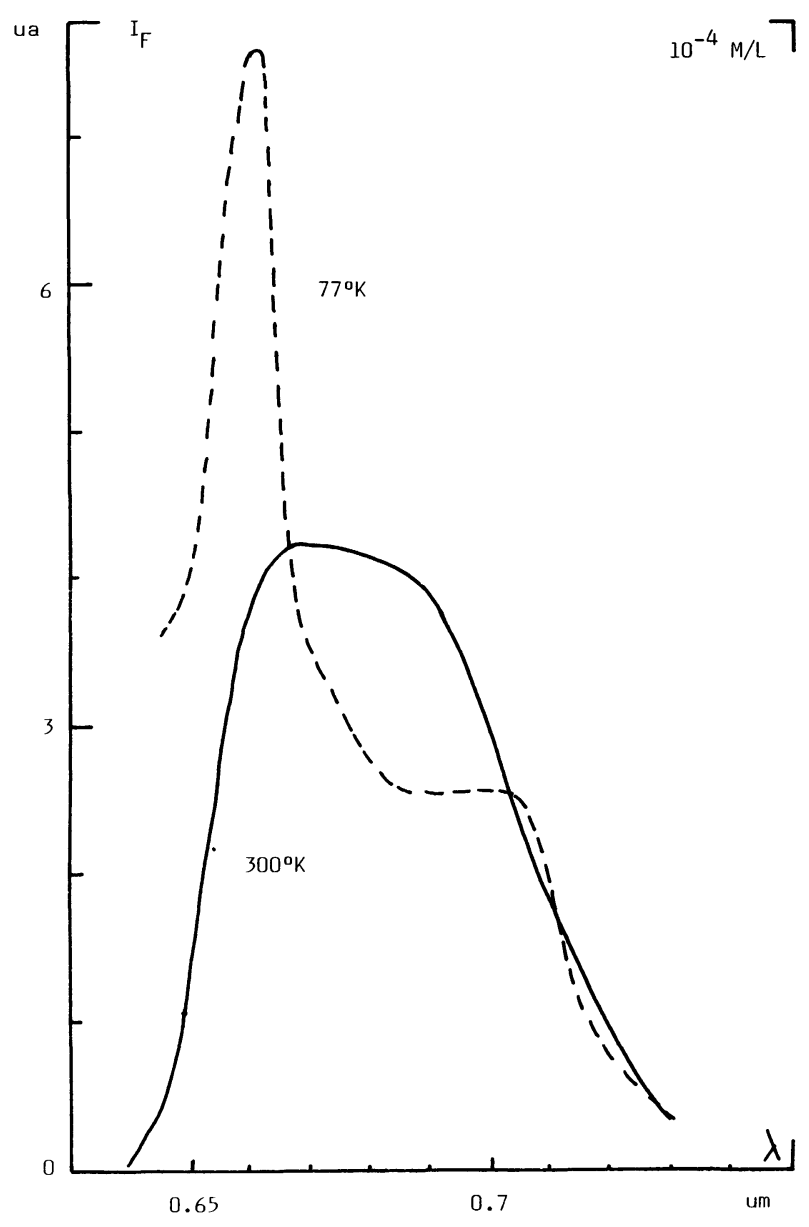

Fig. 2. - Spectres de fluorescence d'une fibre dopée à $10^{-4} \mathrm{M} / 1$ pendant $22 \mathrm{~h}$ : $-300 \mathrm{~K} ;----77 \mathrm{~K}$.

[Fluorescence spectra of $10^{-4} \mathrm{M} / 1$ doped fiber, diffusion time $=22 \mathrm{~h}:-300 \mathrm{~K} ;-\cdots 77 \mathrm{~K}$.] 


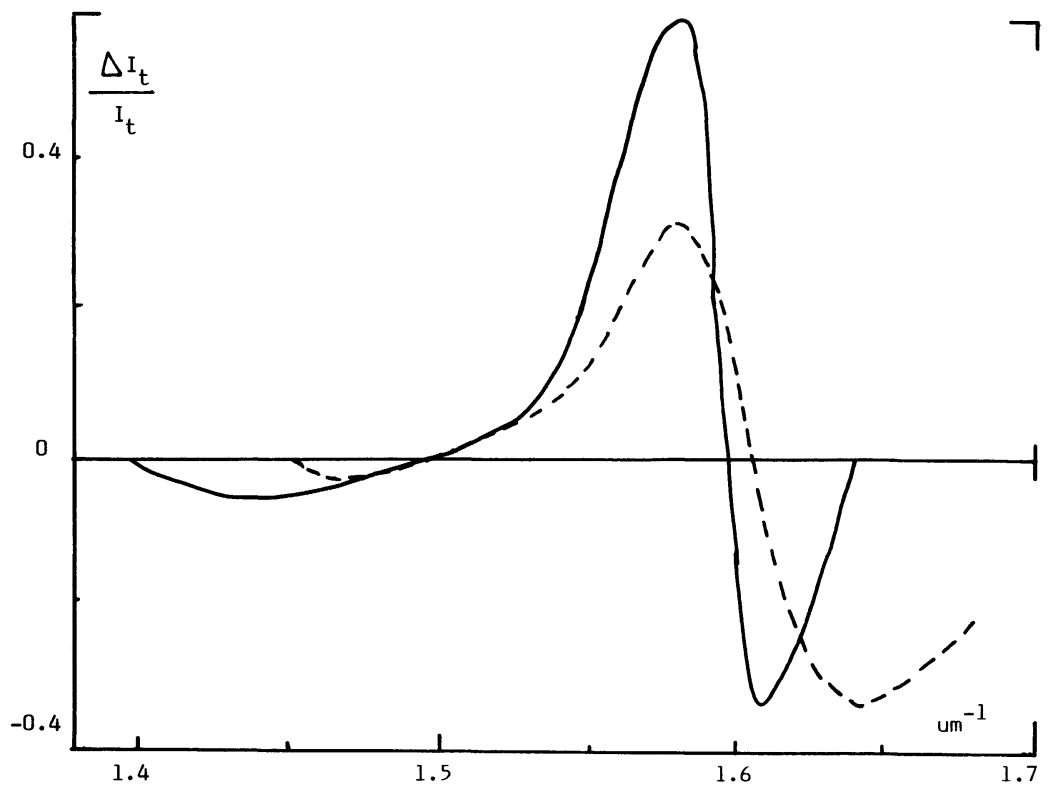

Fig. 3. - Variation relative de l'intensité transmise entre $77 \mathrm{~K}$ et $300 \mathrm{~K}$ pour deux concentrations de Crésyl violet : $10^{-3} \mathrm{M} / \mathrm{l} ;----10^{-4} \mathrm{M} / \mathrm{l}$.

[Transmitted variation intensity for $77 \mathrm{~K}$ and $300 \mathrm{~K}$ temperature and two cresyl violet concentrations : $\left.10^{-3} \mathrm{M} / 1 ;---10^{-4} \mathrm{M} / 1.\right]$

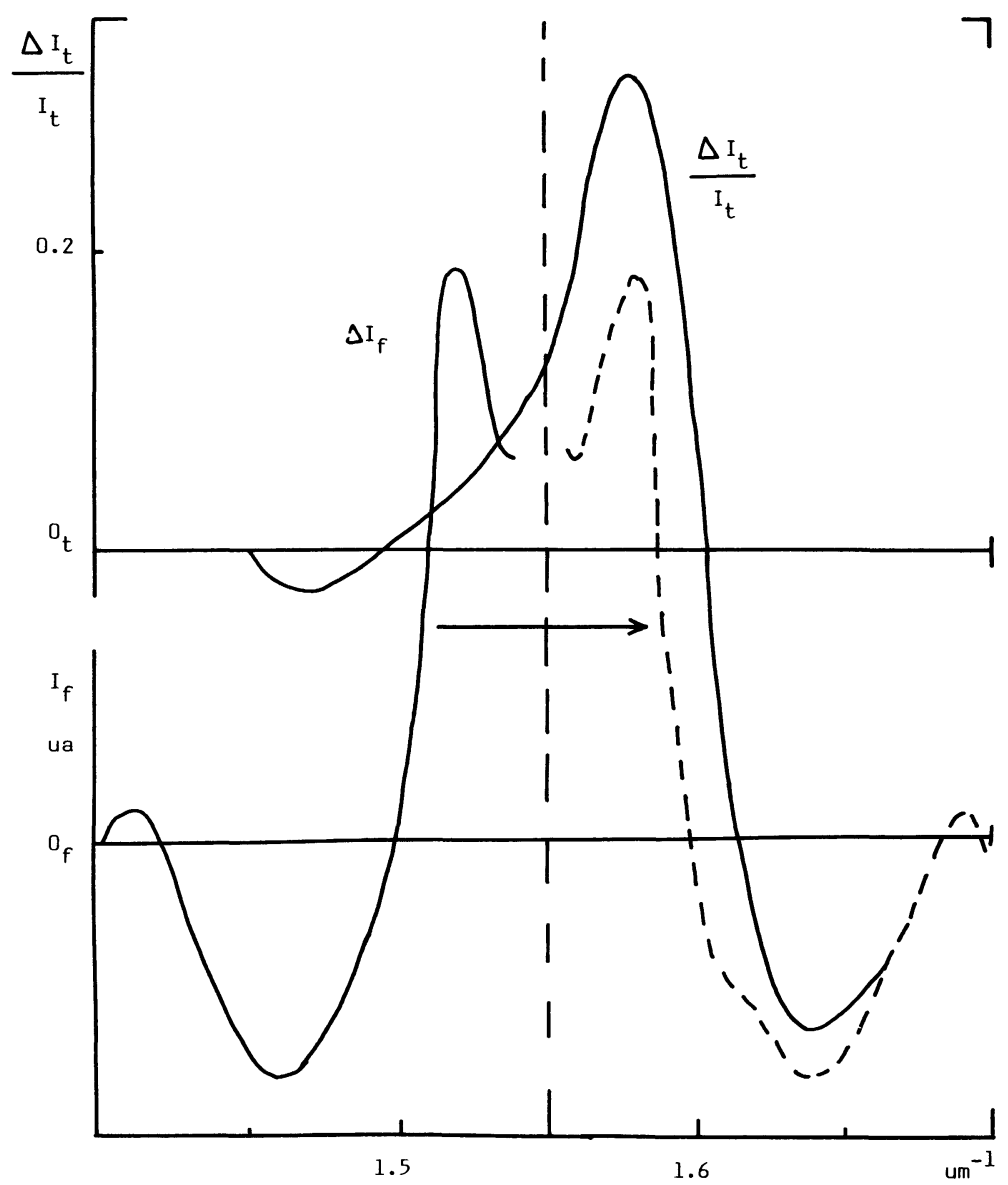

Fig. 4. - Comparaison des spectres de la variation relative d'intensité transmise et de la variation de l'intensité de la fluorescence. --- symétrique de $\Delta I_{\mathrm{f}}$, fibre $10^{-4} \mathrm{M} / \mathrm{l}$.

[Absorption and fluorescence spectra comparison.] 
thermique ces oscillateurs se déplacent avec une vitesse $U$ par rapport au repère fixe lié à la fibre. L'onde lumineuse incidente de nombre d'onde $\sigma_{0}$ dans le repère fixe lié à la fibre possédera un nombre d'onde $\sigma$ pour l'oscillateur en mouvement. La différence $\sigma_{0}-\sigma$ et la vitesse $U$ sont liées par la relation de Doppler $U= \pm\left(\sigma_{0}-\sigma\right) c / \sigma_{0}$. Si les vitesses $U$ sont distribuées suivant une loi du type Maxwell-Boltzman il en résulte un profil spectral gaussien pour le facteur d'absorption.

$$
\alpha=\alpha_{0} \exp -\left[\frac{\sigma-\sigma_{0}}{\gamma}\right]^{2}
$$

avec $\gamma=\sqrt{\frac{2 k T}{M}} \frac{\sigma_{0}}{c}$, où $M$ est la masse de l'oscillateur.

Nous supposerons que sur la largeur à mi-hauteur de la gaussienne, le coefficient $\alpha_{0}$ varie peu avec $\sigma$. La variation de $\alpha$ avec la température est obtenue en différentiant $\alpha$ par rapport à $T$

$$
\begin{aligned}
\Delta \alpha=\left\{\left(\sigma-\sigma_{0}\right)^{2}+\gamma \frac{\Delta \sigma_{0}}{\Delta \gamma}\left(\sigma-\sigma_{0}\right)+\right. \\
\left.\quad+\frac{\gamma^{3}}{2 \alpha_{0}} \frac{\Delta \alpha_{0}}{\Delta \gamma}\right\} \times \frac{2 \Delta \gamma}{\gamma^{3}} \alpha_{0} \mathrm{e}^{-\left(\frac{\sigma-\sigma_{0}}{\gamma}\right)^{2}} .
\end{aligned}
$$

Dans l'expression de $\Delta \alpha$ le facteur multiplicatif de l'exponentielle est un polynôme du second degré en $\sigma-\sigma_{0}$. Pour décrire convenablement les courbes expérimentales, il est nécessaire que ce polynôme admette deux racines réelles et trois extremums. Les conditions ci-dessus seront satisfaites si

$$
\frac{\Delta \alpha_{0}}{\Delta \gamma}<0, \quad \frac{\Delta \sigma_{0}}{\Delta \gamma}>0 .
$$

Le facteur $\gamma$ varie comme $\sigma_{0} T^{1 / 2}$, lorsque $\Delta \sigma_{0} \ll$ $\sigma_{0}$ on peut écrire que $\frac{\Delta \gamma}{\gamma} \approx 0,5 \frac{\Delta T}{T}$, or $\Delta T<0$ nous avons donc $\Delta \alpha_{0}>0$ et $\Delta \sigma_{0}<0$. Sur les spectres expérimentaux l'écart des deux zéros de $\Delta \alpha$ est de $1000 \mathrm{~cm}^{-1}$ pour la fibre à $10^{-3} \mathrm{M} / 1$ de la figure 3 , la différence des deux racines du polynôme en $\sigma$ $\sigma_{0}$ est donc égale à $1000 \mathrm{~cm}^{-1}$, il en résulte que

$$
\frac{\gamma^{3}}{2 \alpha_{0}} \frac{\Delta \alpha_{0}}{\Delta \gamma}=-\left(10^{6}-\gamma^{2}\left(\frac{\Delta \sigma_{0}}{\Delta \gamma}\right)^{2}\right) / 4
$$

avec toutes les grandeurs exprimées en $\mathrm{cm}^{-1}$. En prenant $\Delta \sigma_{0}$ comme paramètre nous avons recherché le meilleur accord entre les spectres théoriques et le spectre expérimental de $\Delta \alpha$. Cet accord est réalisé pour $\Delta \sigma_{0}=-233 \mathrm{~cm}^{-1}$ ce qui nous donne $\Delta \alpha_{0} / \alpha_{0}=0,34$. Le nombre total $N$ d'oscillateurs étant constant le coefficient $\alpha_{0}$ doit vérifier la relation de normalisation

$$
N \Gamma_{0}=\int_{-\infty}^{+\infty} \alpha_{0} \exp -\left[\frac{\sigma-\sigma_{0}}{\gamma}\right]^{2} \mathrm{~d} \sigma
$$

il en résulte que

$$
\alpha_{0}=\sqrt{\frac{M}{4 \pi k T}} \frac{c N}{\sigma_{0}} \Gamma_{0} .
$$

Donc $\Delta \alpha_{0} / \alpha_{0}$ doit être voisin de $0,5 \frac{\Delta T}{T}$ ce qui est voisin de la valeur 0,34 ci-dessus. En ce qui concerne la valeur de $\Delta \sigma_{0}$, il est nécessaire de posséder un modèle donnant la valeur de la fréquence de résonance d'une molécule de colorant placée dans un milieu de constante diélectrique $\varepsilon$ égale à celle du PMMA, la variation de $\varepsilon$ avec $T$ nous donnera la variation de $\sigma_{0}$.

Un modèle de bande à largeur inhomogène permet donc de décrire qualitativement et quantitativement l'évolution avec la température des bandes d'absorption-émission du crésyl violet dans le PMMA. Un abaissement de température affecte les bandes d'émission-absorption dans leur ensemble, une modification de ces bandes sur un petit domaine spectral est possible en éclairant une fibre avec une radiation d'intensité et de largeur spectrale convenablement choisies. L'étude de la transmission en fonction de l'intensité lumineuse devrait nous renseigner sur cette possibilité.

\section{Variation de la transmission avec l'intensité lumi-} neuse.

Une fibre optique en PMMA dopée au crésyl violet par diffusion $\left(10^{-4} \mathrm{M} / \mathrm{l}\right)$ de longueur $1 \mathrm{~m}$ ne transmet pas à la température ambiante la radiation $6328 \AA$ du laser HeNe. Lorsqu'on refroidit cette fibre à la température de l'azote liquide, elle devient transparente à $6328 \AA$, nous pouvons alors l'utiliser pour étudier la transmission de la fibre avec l'intensité lumineuse. Le montage réalisé comprend un jeu d'atténuateurs que l'on peut disposer à volonté avant ou après la fibre de manière à faire varier l'intensité lumineuse dans la fibre tout en maintenant un éclairement du détecteur compatible avec sa linéarité. Un monochromateur nous permet d'isoler la radiation excitatrice de la lumière de fluorescence émergeant de la fibre. Soit $p^{(\mathrm{L})}$ la puissance du laser $\mathrm{HeNe}$, ici $25 \mathrm{~mW}$, la puissance dans la fibre est $p^{(\mathrm{i})}=A_{\mathrm{i}} p^{(\mathrm{L})}$. En sortie de fibre la puissance lumineuse est $p^{(\mathrm{s})}$, sur le détecteur elle sera $p^{(\mathrm{d})}=$ $A_{\mathrm{s}} p^{(\mathrm{s})}$. Le coefficient de transmission de la fibre est $t=p^{(\mathrm{s})} / p^{(\mathrm{i})}=p^{(\mathrm{d})} /\left(A_{\mathrm{s}} A_{\mathrm{i}} p^{(\mathrm{L})}\right)$. L'expérience est réalisée en maintenant le nombre total d'atténuateurs constant, $A_{\mathrm{s}} A_{\mathrm{i}} p^{(\mathrm{L})}$ est donc une constante et $p^{(\mathrm{d})}$ représente une mesure de la transmission de la fibre. La figure 5 représente la variation de $t$ en fonction de la puissance lumineuse dans la fibre pour une fibre dopée et une fibre vierge. La fibre dopée présente une variation linéaire du coefficient de transmission qui n'existe pas dans la fibre vierge, cette variation résulte donc de la présence du 


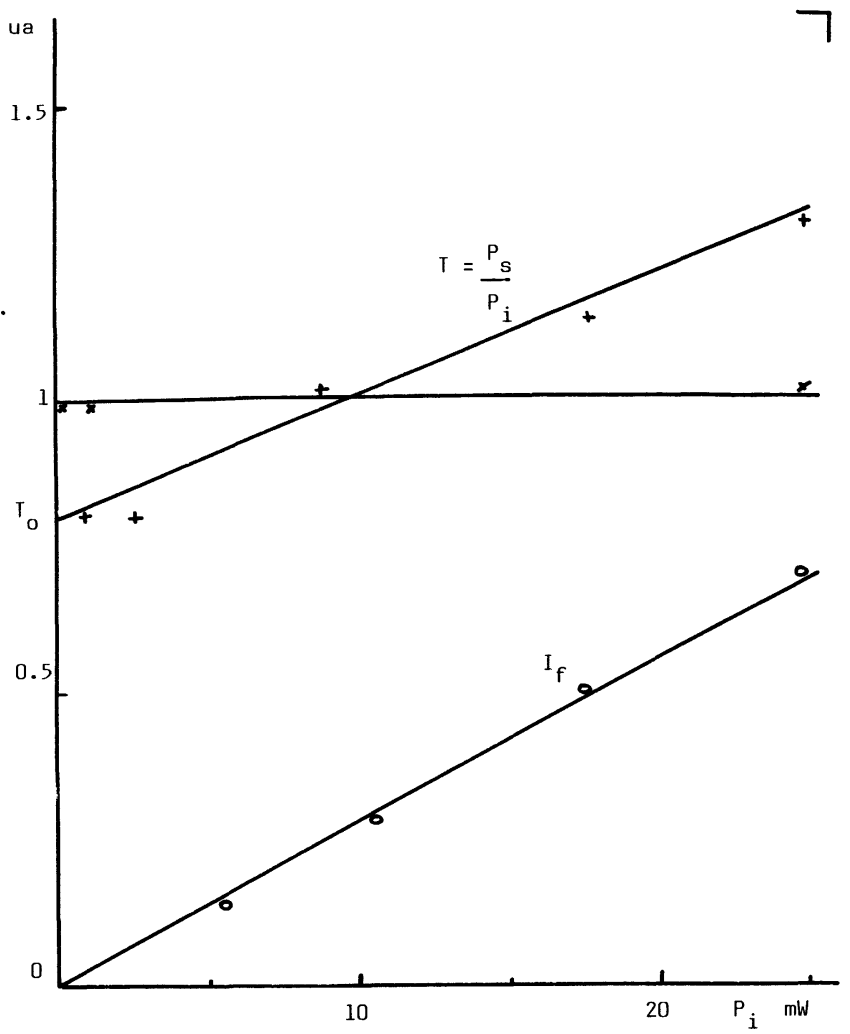

Fig. 5. - Evolution de la transmission et de l'intensité de la fluorescence à $6600 \AA$ avec la puissance lumineuse incidente $:+$ fibre dopée $; \times$ fibre vierge ; O fluorescence.

[Transmission at $6328 \AA$ and fluorescence at $6600 \AA$ versus light intensity : + doped fiber ; $\times$ non doped fiber ; O fluorescence.]

colorant dans la fibre. Par ailleurs, nous avons constaté que l'intensité de la lumière de fluorescence est proportionnelle à l'intensité excitatrice. Pour interpréter ces résultats nous supposerons que les molécules de colorant sont assimilables à des systèmes à deux niveaux, les niveaux singlets $S_{0}$ et $S_{1}$ de cette molécule. Soient $N_{0}$ la concentration des molécules, $N$ la population du niveau $S_{1}$ alors:

$$
\begin{aligned}
& \frac{\partial N}{\partial t}=\left(N_{0}-2 N\right) \sigma I-\frac{N}{\tau}, \\
& \frac{\partial I}{\partial s}=-\sigma\left(N_{0}-2 N\right) I
\end{aligned}
$$

où $\sigma$ est la section efficace d'absorption des molécules, $\tau$ le temps de désexcitation du niveau $S_{1}$ et $I$ le nombre de photons par seconde et unité de surface [4]. La lumière se propageant dans une fibre très multimode, il est possible de choisir un modèle de rayons paraxiaux pour décrire la propagation, la variable sera l'abscisse curviligne $s$ d'un point sur le rayon lumineux [5].

Pour un régime stationnaire, et une intensité $I$ pas trop grande $2 N$ est petit devant $N_{0}$, dans ce cas :

$$
N \approx \sigma \tau N_{0} I, \frac{\partial I}{\partial s}=-\sigma N_{0} I+2 \sigma^{2} \tau N_{0} I^{2} .
$$

Dans l'expression de $N$ nous ne conservons pas le terme en $I^{2}$ car il dépend de $\tau^{2}$ qui est très petit, par contre dans l'expression de $I$, ce terme ne dépend que de $\tau$. L'équation différentielle satisfaite par $I$ peut être généralisée sous la forme :

$$
\frac{\mathrm{d} I}{\mathrm{~d} s}=-\alpha I+\beta I^{2}
$$

avec $\beta / \alpha$ indépendant de $s$.

La résolution de cette équation nous donne le facteur de transmission de la fibre $t=I / I_{0}$ en fonction de l'intensité incidente $I_{0}$

$$
\begin{gathered}
t=\frac{\mathrm{e}^{-\gamma L}}{1-\frac{\beta}{\alpha} I_{0}\left(1-\mathrm{e}^{-\gamma L}\right)} \\
\gamma=\frac{1}{a \cos \Theta} \int_{0}^{a} \alpha \mathrm{d} r
\end{gathered}
$$

$\Theta=$ inclinaison du rayon lumineux sur l'axe de la fibre,

$a$ = rayon du cœur de la fibre.

Le deuxième terme du dénominateur de $t$ est petit nous pouvons donc utiliser un développement limité de $t$.

$$
t \approx \mathrm{e}^{-\gamma L}\left\{1+\frac{\beta}{\alpha} I_{0}\left(1-\mathrm{e}^{-\gamma L}\right)\right\}
$$

Pour $I_{0}$ petit $t \approx \mathrm{e}^{-\gamma L}$ que nous poserons égal à $t_{0}$ donc

$$
\frac{t-t_{0}}{t_{0}} \approx \frac{\beta}{\alpha} I_{0}\left(1-\mathrm{e}^{-\gamma L}\right) .
$$

Comme il est d'usage pour les fibres optiques définissons une longueur effective et une surface effective de la fibre par les relations :

$$
L_{\text {eff }}=\frac{1-\mathrm{e}^{-\gamma L}}{\gamma} \frac{1}{a \alpha} \int_{0}^{a} \alpha \mathrm{d} r, S_{\text {eff }}=S \cos \Theta
$$

dans ce cas :

$$
\frac{\Delta t}{t_{0}}=\beta L_{\text {eff }} \frac{P}{S_{\text {eff }}} \cdot \frac{1}{h \nu}
$$

$P$ est la puissance lumineuse du laser.

A partir de cette relation il est possible de calculer $\beta$ par la relation :

$$
\beta=\frac{\frac{\Delta t}{t_{0}} S_{\mathrm{eff}} h \nu}{L_{\mathrm{eff}} P}
$$

avec $\frac{\Delta t}{t_{0}}=0,63, L=10^{2} \mathrm{~cm}, \quad d=250 \mu \mathrm{m}, \quad \lambda=$ $0,63 \mu \mathrm{m}$ et $P=25 \mathrm{~mW}$ nous obtenons :

$$
\beta_{\text {exp }}=0,4 \times 10^{-22}\left(\frac{S_{\text {eff }}}{S} \cdot \frac{L}{L_{\text {eff }}}\right) \mathrm{cm} \mathrm{W}^{-1} .
$$


La valeur théorique de $\beta$ est $\beta_{\text {th }}=2 N_{0} \sigma^{2} \tau$. La fibre que nous avons utilisée a été dopée à l'aide d'une solution de crésyl violet à $10^{-4} \mathrm{M} / 1$ dans du méthanol pendant $22 \mathrm{~h}$, nous admettrons que la valeur de $N_{0}$ est la même que celle de la solution soit $6 \times 10^{16} \mathrm{~cm}^{-3}$. Pour $\sigma$ et $\tau$ nous n'avons pas les données exactes pour le crésyl violet à $77 \mathrm{~K}$, avec $\sigma=3 \times 10^{-16} \mathrm{~cm}^{2}$ et $\tau=10^{-9} \mathrm{~s}$ nous obtenons $\beta_{\text {th }}=0,10810^{-22} \mathrm{~cm} \mathrm{~W}^{-1}$ ce qui est voisin de la valeur expérimentale. Ces valeurs de $\sigma$ et $\tau$ sont supérieures à celles correspondant à une solution liquide de rhodamine $\left(\sigma \sim 10^{-16} \mathrm{~cm}^{2}, \tau \sim 0,110^{-9} \mathrm{~s}\right)$ [6]. L'insertion du colorant dans la matrice solide provoque une augmentation de $\sigma$ et $\tau$, des mesures plus précises sont encore nécessaires pour vérifier cette hypothèse.

Par ailleurs, le modèle ci-dessus prévoit une variation linéaire de l'intensité de la fluorescence avec la puissance lumineuse excitatrice ce qui est conforme à l'expérience.

Cette étude confirme donc qu'il est possible d'obtenir un comportement non linéaire d'une fibre optique en polymère dopée avec un colorant ceci à partir d'une source lumineuse de puissance relativement modeste.

\section{Conclusion}

Une modification des bandes d'absorption-émission du crésyl violet diffusé dans le PMMA a été mise en évidence lors du passage de la température ambiante à celle de l'azote liquide. Le maximum d'absorption se déplace de $233 \mathrm{~cm}^{-1}$ avec une variation d'amplitude de $34 \%$; le maximum de la fluorescence se déplace vers les courtes longueurs d'onde. Par ailleurs, à la température de l'azote liquide nous avons observé une augmentation de la transmission de la fibre avec l'intensité lumineuse.

L'originalité des propriétés optiques du PMMA dopé avec un colorant, le confinement de la lumière apporté par la fibre optique, conduisent donc à la réalisation d'un composant optique intéressant pour l'ingénierie optique (capteur, absorbant saturable...).

Les prolongements de cette étude liés à l'utilisation de températures plus basses (He liquide), de puissance lumineuse plus grande (laser déclenché) devraient nous conduire à des données nouvelles sur le comportement des fibres en polymère chargé de colorant.

\section{Bibliographie}

[1] Gromov, D. A., Dyumavev, K. M., Manenkov, A. A., Maslyukov, A. P., Matyushin, G. A., Nechitailo, V. S. et Prokhorov, A. M., J. Opt. Soc. Am. B 2 (1985) n 7.

[2] Batchelder, J. S., Zewail, A. H. et Cole, T., Appl. Opt. 20 (1981) 3733-3754.
[3] SAISsY, A. et CAI, Z. P., Huitièmes Journées Nationales d'Optique Guidée, Montpellier (1987).

[4] Gires, F. et Combaud, F., J. Phys. France 26 (1965) 325-330.

[5] SNyder, A. W. et Love, J. D., Optical Waveguide Theory.

[6] Penzofer, A., Appl. Phys. B 40 (1986) 85-93. 